\title{
Current Trends in MEMS Drug Delivery Techniques
}

\author{
Mohammad Sibgat Ullah ${ }^{1}$, Rishab Kaw ${ }^{2}$, Peer Athar Rasool ${ }^{3}$, Neeraj Tripathi ${ }^{4}$, Vikram Singh ${ }^{5}$ \\ Department of Electronics and Communication Engineering, \\ Shri Mata Vaishno Devi University, Katra, J \& K, India - 182320 \\ 12013eec26@smvdu.ac.in \\ 22013eec36@smvdu.ac.in \\ 32013eec30@smvdu.ac.in \\ ${ }^{4}$ neeraj.tripathi@smvdu.ac.in \\ 5vikram.singh@smvdu.ac.in
}

\begin{abstract}
The popularisation of the microsystems has paved a way for the evolution of a new class of controlled drug delivery devices which allow tailored delivery of drugs. Additionally, these drug delivery systems enhance efficiency and patient consent. Developing better drug delivery methods is the prime target of the pharmaceutical companies worldwide. The process of drug delivery is as essential as the drug's activity in deciding its effectiveness. These drug delivery systems do not simply release the drug but disseminate the drug in a peculiar manner in accordance to which it has been engineered. Although in their initial stages, the microelectromechanical systems (MEMS) demonstrate the immense potential for conquering the various challenges that are faced by the present drug delivery techniques. Microneedles, micropumps, microvalves, and implantable drug delivery systems have been developed using microfabrication techniques. Several microdevices have been engineered such that they deliver multiple drugs with different dosages in series or parallel which provide several advantages such as extension in the variety of the desirable compounds and precise dosing. Transdermal drug delivery through microneedles enables enhanced permeation through skin layers into the body. Multilayer patch systems lead to an excellent approach in the oral drug delivery. This review examines various MEMS drug delivery techniques along with their diverse benefits.
\end{abstract}

Keyword- Drug Delivery, Microneedles, Microvalves, Micropumps, Multilayer Patch

\section{INTRODUCTION}

Enhancement of microfabrication techniques has given rise to the creation of new kind of devices which have microscopic size and can be used to carry out different electrical and mechanical functions, such systems are known as micro electro-mechanical systems (MEMS). These can be highly applied for controlled therapeutic delivery leading to the versatile advancements in the process of drug delivery. These micro electro-mechanical systems can be employed in diverse ways in order to carry out the process of drug delivery and enhance efficiency, convenience and patient compliance. The process of drug delivery is a very important activity as it readily effects the process of treatment of patients and directly relates to their healing ability. So, worldwide, a lot of investments and researches are done to enhance the drug delivery techniques. The use of micro electromechanical systems for carrying out the process of drug delivery truly enhances this mechanism and leads to higher therapeutic benefits. MEMS techniques have permitted the evolution of miniaturised diagnostic tools which provide higher efficiency in the process of drug delivery [1]. These drug delivery systems developed by MEMS techniques enable controlled drug release kinetics, thus, preventing the wastage of drugs and reducing their side-effects. Such systems can be highly utilised in the treatment of diabetes wherein the requirement of pulsatile release of insulin is required [2].

MEMS technology has led to the creation of various microfabricated devices which can be utilised in numerous ways to achieve the proper delivery of sedatives. Such devices include multilayer micropatches, micropumps, microneedles and microvalves. These devices can deliver multiple drugs with precise dosages simultaneously which increases the effectiveness of drug delivery. Micropatches can be used in oral drug delivery for the sake of achieving excellence in the distribution of drugs in the gastrointestinal track leading to better healing effects. The transdermal drug delivery can be enhanced by the usage of microneedles which enable targeted drug delivery and prevent the sense of discomfort in patients by reducing the pain which may occur with conventional transdermal delivery of drugs. The Micropumps and Microvalves are adopted for the development of various implantable drug delivery systems which can duly transform the process of drug delivery to higher grounds and provide immense patient compliance. Such systems are highly beneficial due to their potential for assimilation with microelectronics [3]. 


\section{TRAnSDERmal DRUg DeliVery}

Conventional transdermal drug administration is severely limited by molecular weight of drugs due to the skin's low permeability. The top epidermal layer of the skin, the stratum corneum is the skin's primary permeation barrier and limits the diffusion of drug molecules into the skin. It's about 10-20 $\mu \mathrm{m}$ thick dead tissue. Skin nerves are situated 100-200 microns below them [4], [5]. Microneedles increase the permeability of the skin by creating micro-paths across the stratum corneum for delivering drugs [1], [6].

Microneedles are needles of micron-scale fabricated using MEMS technology and are used for transdermal drug delivery [7]. MEMS technology is needed to precisely manufacture arrays of these microneedles which are approximately of $100 \mu \mathrm{m}$ length. They should be of robust nature so that they penetrate this layer of skin, as well as their length should also be short enough to avoid pain by stimulation of nerves in deeper tissues [4], [8]. Microneedles allow localised and painless delivery of drugs to targeted tissues thus reducing the side effects and minimising the dose of drug delivered [9].

A wide variety of microneedles are designed for this purpose. These allow different approaches of drug delivery. They are categorised as:

\section{A. Solid Durable Microneedles}

This is the simplest microneedle array system that produces micropunctures in the stratum corneum and hence renders the skin more permeable. Drugs can be delivered by this type of array in two ways.

- Application of drugs by a patch on the micro-paths that remain after microneedle removal [6], [10], [11] (See Fig. 1(a)).

- Drugs are first coated onto the solid microneedles and then microneedle patch is applied on the skin. This drug coating dissolves after insertion into the skin. This microneedle patch can be thrown away or reused after reapplication of the drug on the microneedles [12], [13] (See Fig. 1(b)).

\section{B. Solid Degradable Microneedles}

In this type, entire needles are manufactured from drug infused biodegradable and water-soluble polymers. This maximises the drug volume capacity of microneedles however their fabrication is more difficult [14] (See Fig. 1(c)).

\section{Hollow Microneedles}

This type of microneedle array system has bores on tips of microneedles that are connected to drug reservoirs. They work in a similar fashion as conventional hypodermic injections. These puncture the skin and then infuse the drugs that are in a liquid formulation through the needle bores into the tissue. These do not have the drug capacity limitation as in the case of solid needles and arbitrary quantities of drugs in liquid formulation can be used [15], [16], [17]. However, they pose the danger of breaking off in the skin as they are hollow and less structurally stable than solid microneedles [18] (See Fig. 1(d)).

\section{ORAL DRUG DELIVERY}

The oral route is the most common route chosen for drug delivery. Right from emerging of the medical sciences, a lot of work has been conducted to develop different pills and capsules, fed through the oral route to the patients, so as to cure them of various diseases and disorders. But, still we are unable to attain precise and proper dosage of drugs through this route and hence a lot of drugs are unable to completely cure the patient because of the various barriers such as stomach ph, mucus, and enzymes associated with the gastrointestinal track (See Fig. 2).

The use of MEMS (Micro Electro Mechanical System) techniques provides a significant way to overcome such barriers and deliver drugs to the patients in a defined manner to improve their therapeutic benefits and reduce their side effects.

Besides drug delivery, MEMS techniques can be used to develop in vitro gastrointestinal track models that can help to improve drug delivery [1]. The gastrointestinal track also known as mucosal track provides immense opportunities for proper drug delivery, especially, for peptides and proteins. By preventing the degradation in the gastrointestinal track we can improve the bioavailability of the drugs. The main reason behind improper therapeutic benefits from oral drug delivery is the presence of enzymes in the gastrointestinal track and limited permeability of the epithelial barrier. These obstacles can be abolished by using appropriate knowledge of surface chemistry with the microfabrication technology [19], [20].

The solutions offered by MEMS technology provides control over the drug release rate and hence, can make the delivery kinetics more adaptive to therapeutic benefits. Besides, such technology can be used for effective multiple drug administration. The geometry of such systems can be exploited for delivery of drugs in a particular direction, thus, directing the release of drugs towards the walls of the gastrointestinal track rather than the lumen so that the drug transport can be made much more effective [21], [22]. 

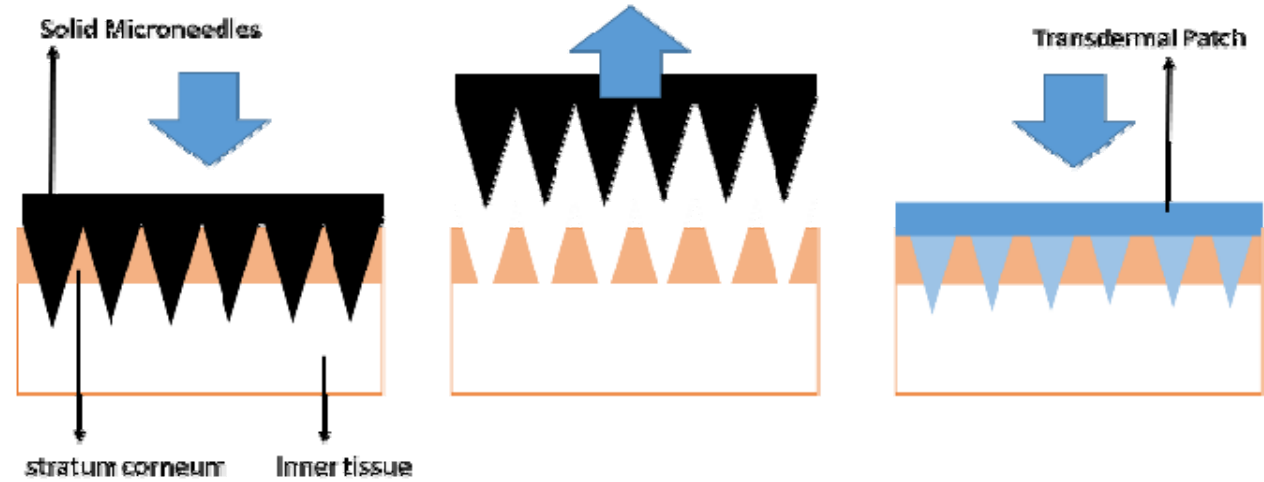

(a) Micropores created using solid microneed les, followed by application of drug using transdermal patch
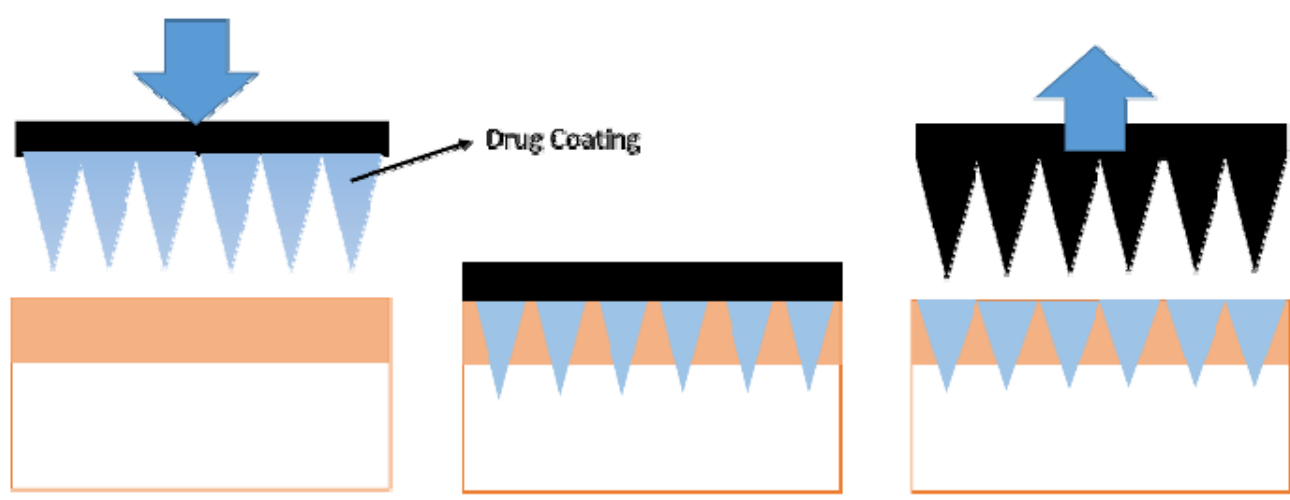

(b) Solid microneed les coated with drug

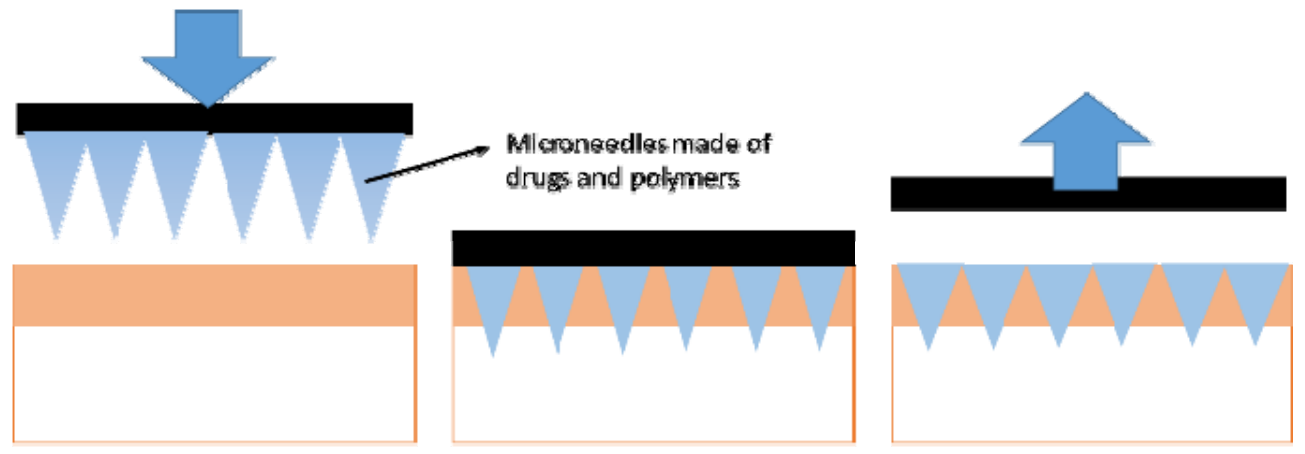

(c) Solid microneedles made of drugs and polymers, which remain in skin and dissolve orer time to deliver drugs

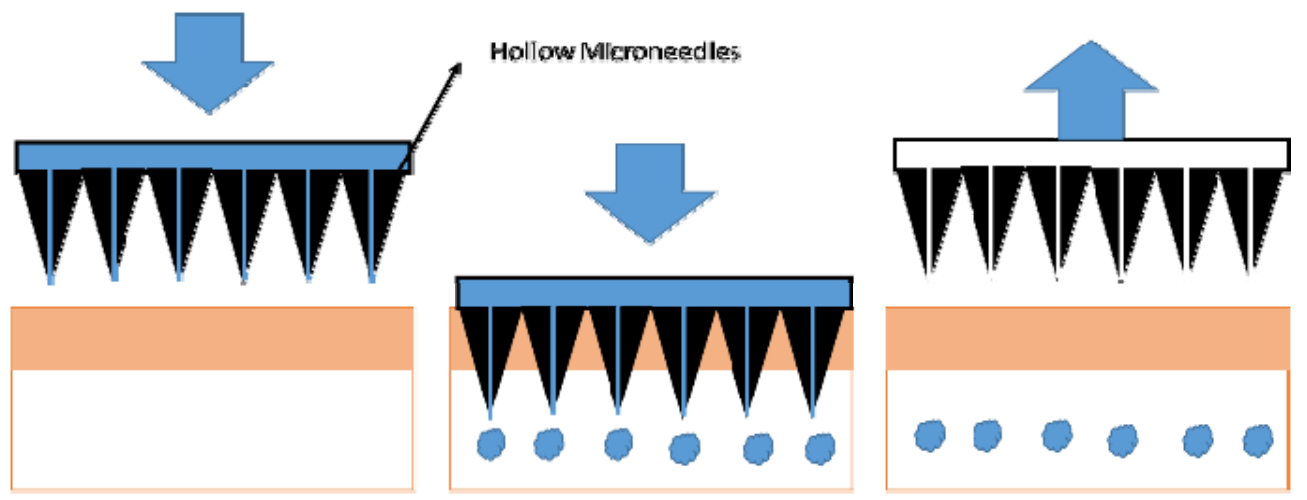

(d) Hollow microneedles used for continuousdrug delivery via drug reservoirs

Fig. 1. Different methods of drug delivery using microneedles (Adapted from [50]). 
Due to the usage of these devices in oral drug delivery, this field attains numerous improvements. Microsystems for oral drug delivery are designed in such a manner so as to maximise their residence time in the gastrointestinal track and provide unidirectional release of drugs towards the intestinal epithelium and also regulate the release of drugs in a sustained manner. These properties make such devices important for not only improving the drug permeation but also improving its solubility and causing the degradation of drugs in such a way which makes them highly effective [23], [24]. Hence, the use of microdevices for oral drug delivery improves this mechanism by leaps and bounds and abolishes most of the barriers associated with oral drug delivery.

Microfabricated multilayer patches for oral drug delivery enables better absorption and stability of drugs in the intestinal epithelium. These patch systems are designed with thin, flexible rate controlling membrane, a drug reservoir, an impervious backing and an adhesive. These patches stick to the intestinal villi. They cause the controlled flow of drugs from the drug reservoirs present on these patches. In this way, the movement of drugs is properly regulated into the blood stream, thus, preventing any harsh effects of the drugs on the patient and hence such systems lead to the improvement in the patient compliance.

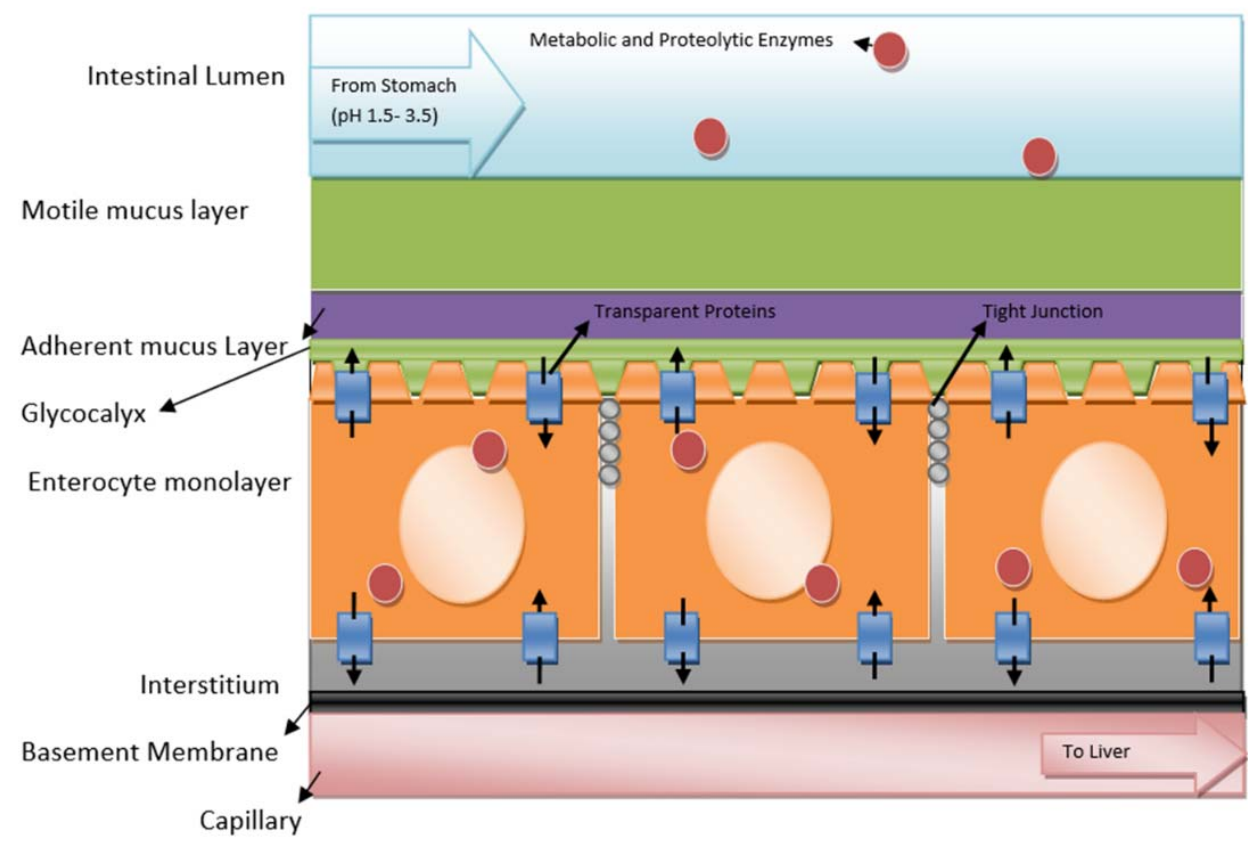

Fig. 2. Physiological barriers to oral drug delivery (Adapted from [23]).

Microfabricated patches for oral drug delivery are usually associated with important roles which are given as:

(i) Bioadhesion with the intestinal epithelium for retention of dosage form,

(ii) Precisely controlled release of drugs to improve its effectiveness and prevent any side effects of these drugs,

(iii) Release of drugs in the direction of the intestinal epithelium to improve the efficacy of dosage preventing the wastage of drugs and improving their therapeutic benefits [25].

\section{A. Micropatch}

Orally delivered particles are meant to transmit through the gastrointestinal track. If the size of these particles is large, they may get cornered in the mucus layer covering the epithelium providing their concise residence inside the gastrointestinal track. So, various micro and nanoparticles have been developed which can penetrate the epithelium but their uptake is restricted to Peyer's patches (See Fig. 3). Also, such particles are associated with a rise in poisonous accumulation and poor biodistribution [25], [26], [27].

Therefore, in order to overcome such hindrances, microfabricated patch systems are developed using the MEMS technology which acts as an alternative to the particulate drug delivery. These micro patches are easily able to transit between the intestinal villi and are not consumed by the cells because of their wider size. Micro patches are thin and flat so that maximum area of these patches can come in contact with the intestinal villi. Besides, due to their flat design, very less amount of area of these patches achieves contact with the liquids flowing through the intestine hence, preventing such devices from releasing drugs into the intestinal fluids rather than towards the intestinal villi [1]. 
Micro patches are associated with multiple drug reservoirs loaded with various drugs. These reservoirs allow the unidirectional release of drugs, thus, enhancing their efficiency. In order to target a particular location in the gastrointestinal track, micro patches can be designed in such a manner that they get stuck to the particular location while they travel through the gastrointestinal track and in this way specific cell targeting can be achieved using micro patches. In micro patches by doing some surface modifications on the reservoir side, drugs are able to take the shortest diffusional path in order to reach the intestinal epithelium [26], [27].

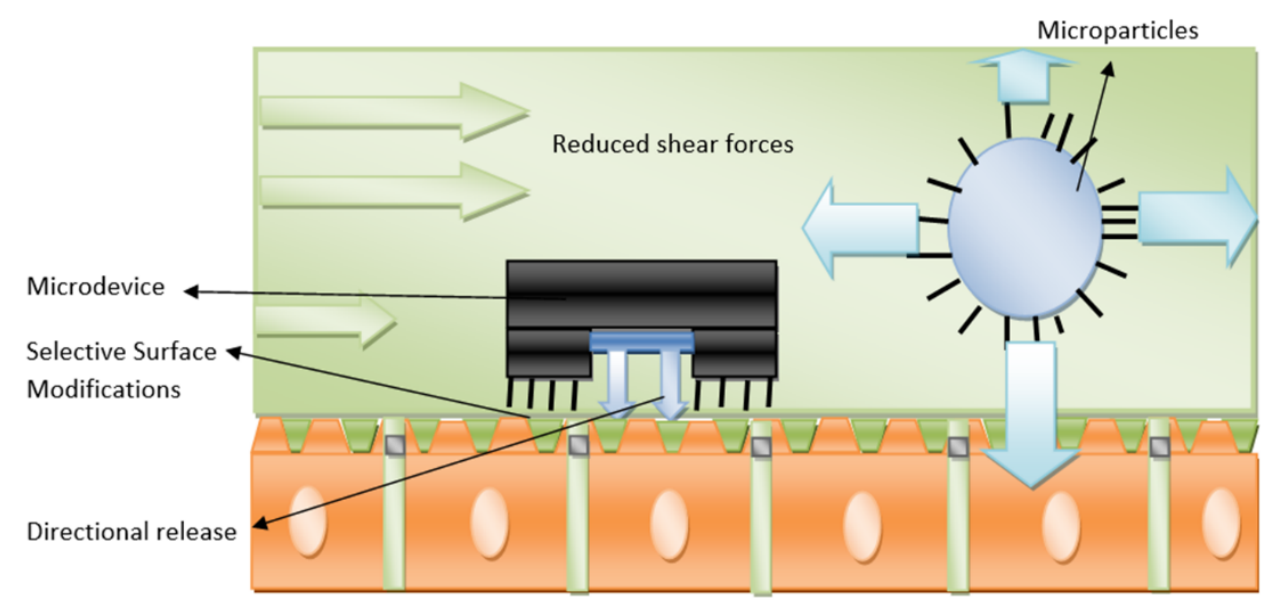

Fig. 3. Advantages offered by microdevices for oral drug delivery (Adapted from [23]).

In micro patches, due to the direct contact between the micro patches and the intestinal epithelium, there is no need for usage of excipients which would help in the dissolution of drugs. By using different polymeric materials for making various reservoirs on the micro patches for storing different drugs, the time and rate of release of drugs can be precisely regulated as different polymeric materials have different dissolution rates and hence, we can achieve a controlled release of several drugs leading to better therapeutic effects and also preventing the wastage of drugs and reducing their side effects [28], [29].

Silicon and silicon dioxide are not biocompatible materials, so they are not used in the fabrication of oral drug delivery systems. Hence, in search for finding out the best material for developing of such systems, polymers opt to be one of the best options.

Polymers are considered to be one of the most economical substrates for fabrication of micro patches. Injection moulding and embossing are utilised in the fabrication of microdevices. Polymers are mainly modified through various photolithographic techniques. Patterning of photolithographic polymers - SU-8 and PMMA is done using micro-molding. The availability of various polymeric materials for microfabrication provides flexibility to the designer, as the designer can design the microdevices with proper drug release kinetics, biodegradability, cell absorption and various other phenomena related to micropatches by using these materials appropriately [28], [29].

The usage of such materials makes microdevices biocompatible and hence, improving the safety concerns associated with the usage of such devices. Photolithography can be also used for filling of Micropatch reservoirs. The materials such as poly(ethyl glycol) dimethycrylate (PEGDMA) is used for such process. Such photolithography also includes exposing the substrate to the UV light which results in filling of microdevices with drugs for sequential delivery of therapeutics [30]. Besides, these micro-reservoirs can also be filled with drugs using microinjection techniques.

\section{B. Bioadhesion}

The main requirement of micropatches is bioadhesion with the intestinal epithelium which enhances the residence time of the device inside the gastrointestinal track and thus improves the drug absorption process. Due to the use of mucoadhesive systems, the drug concentration gradient at the specific site gets enhanced because of the intense contact.

\section{1) Bioadhesion Chemical approach}

Mucoadhesion process is implemented through following steps: First, intimate contact with the tissue is made by wetting and swelling of the polymer, then, entanglement between the polymer and the mucin chain is achieved and hence, weak chemical bonds start to form attaching the mucoadhesive device to the site [31], [32]. Another chemical approach involves the use of lectin as a target molecule to enhance adhesion. The microdevices modified with tomato lectin are found to remain bound to the target over various consecutive 
washes, thus, leading to highly efficient targeted drug delivery. Microspheres modified with lectin can also be used for drug delivery but they appear to be less stable when associated with consecutive washes. Micropatches are more stable in such environment because of their flat shape and larger contact area. Abundant research has been done for improving the stability of micropatches in the intestinal track so as to enhance drug delivery [25], [26], [27].

\section{2) Bioadhesion Physical approach}

Various modifications in the design of microdevices can be made so that these devices can adhere easily at the specific site. One such modification involves the designing of precisely shaped microneedles and microposts on micropatches which improves their bioadhesion [1]. Microneedles which are mainly developed for transdermal drug delivery have been improved a lot and made biocompatible by designing such microneedles with materials such as poly(lactic acid), poly(glycolic acid) and PLGA [33], [34]. Besides, these needles have also been improved to work efficiently in wet environments. Such microneedles or microposts can be highly utilised in oral drug delivery to improve bioadhesion of the devices. By using such microneedles with the micropatches retention to the intestinal villi can be highly improved. Besides, these microposts can also be used to regulate the flow of drugs mechanically [25], [26], [27].

In addition to this, nanostructures can be used to improve the bioadhesion of microstructures. Due to these nanostructures, van der walls forces can come into existence which leads to stiction of microdevices in the intestinal track at specific sites. As the number of nano-adhesive elements increases, the surface area to volume ratio of these nanostructures rises leading to a prominent growth in the van der walls forces [35], [36]. Hence, by using nanostructures we can improve the bioadhesion by simply bringing some changes in the geometry of these structures. These nanostructures such as nanowires are synthesised and developed on micropatches using "liquid solid" method [37], [38]. Hence, use of such nanostructures can drastically improve the process of bioadhesion inside the intestinal track.

However, most of the microdevices are made more bioadhesive by using physical methods such as nanowires modified chemically which improves their mucoadhesive properties and leads to proper stiction at specified sites thus, improving the process of oral drug delivery and hence, leading to better therapeutic effects.

\section{IMPLANTABLE DEVICES FOR DRUg DELIVERY}

Recent advances in MEMS technology have paved way for significant advancements in the development of implantable MEMS devices for drug delivery. The advantages offered by MEMS which include the small size of devices, low power consumption has inspired the development of implantable devices which can be placed inside a human body. They can release drugs of different dosage and at the same time, the drug delivery pattern and the duration can be controlled. Moreover, these devices have a simple release mechanism and very accurate dosing. With such devices, targeted drug delivery with improved efficacy can be achieved while reducing the side-effects. Implantable MEMS devices are ideal for localised and precise drug delivery. The size of these devices is in micrometers hence they can be implanted in a specific region of the body for localised drug delivery. The sensors used can constantly keep track of the drug release pattern and thereby provide useful information for further processing. The implantable MEMS devices provide a strong alternative for treating chronic diseases. These devices have sensors and electronic circuitry and can be placed inside a human body for a long time while working properly.

The main design concerns for implantable devices are applied voltage, power consumption, dosing accuracy, concentration, frequency, drug interaction and toxicity. Implantable devices are designed for applications - in vivo and in vitro [39], [40]. The current trends in the technology which focus on developing a wireless system for energy and data transmission facilitate the in vivo application of these devices. Further, the implantable devices can be designed for both active and/or passive drug delivery. The active devices are reservoir-based drug delivery devices; they contain an actuator due which the device is able to perform active operation [41]. Active devices can be refillable which eliminates the need for a very large reservoir moreover active devices can be tuned according to the particular need of the patient [40]. Passive devices release drugs in a more controlled way and they rely on diffusion process for release of the drug.

Since these devices are implanted inside the human body or kept under the skin, therefore, one of the greatest issues with such devices is their biocompatibility. The impact of such devices on the immune response is a very important factor to be considered. The implanted device in the human body can attract an attack from the immune system which may treat it as an external intruder. Thus, with time, these devices may not be able to deliver the expected performance; in fact, they may be reduced to malfunction [42]. For these reasons the biocompatibility of MEMS materials, which are used to design such implantable devices, needs to be evaluated with greater care and precision. As of now some of the materials which have proven to have good biocompatibility include Poly Methyl Methacrylate (PMMA), poly-Dimethylsiloxane (PDMS), SU-8 photoresist and parylene C [43]. 
Two very significant and successfully tested implantable devices are micropumps and microvalves.

\section{A. Micropumps}

Micropumps are used for active drug delivery of fluids from a reservoir to dispense therapeutic agents into human body [39] (See Fig. 4). For micropumps, high volumetric flow rate and high resolution are two factors of significant interest [42]. The main limitations associated with micropumps are their low reliability, high power consumption and low delivery rates. Micropumps are broadly divided into two categories which are mechanical micropumps and non-mechanical micropumps [44].

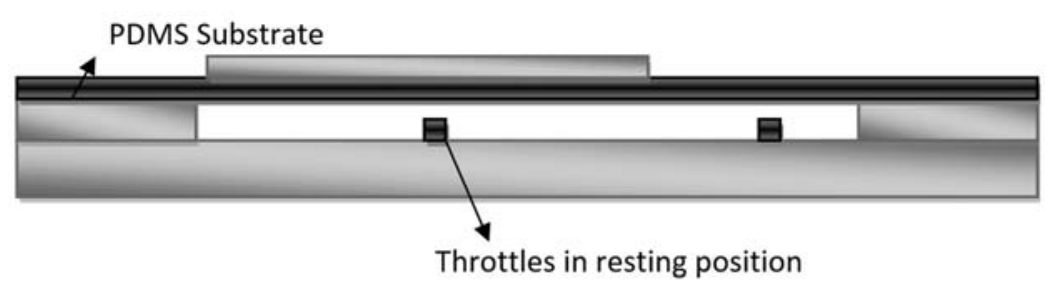

(a) Unenergized

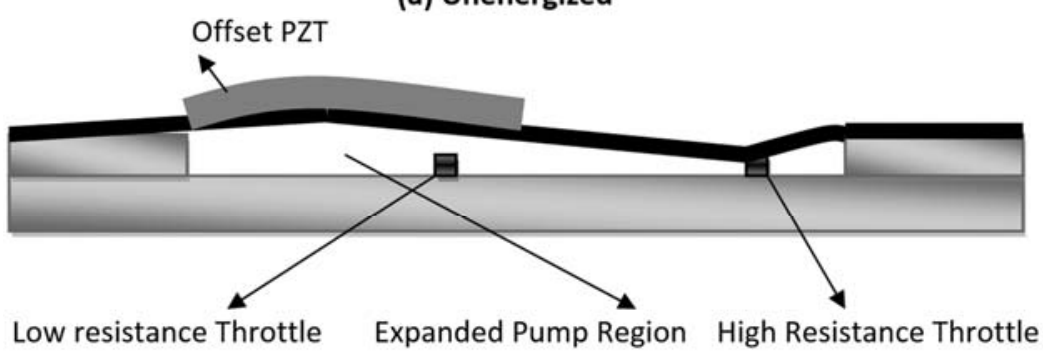

(b) Suction Stroke

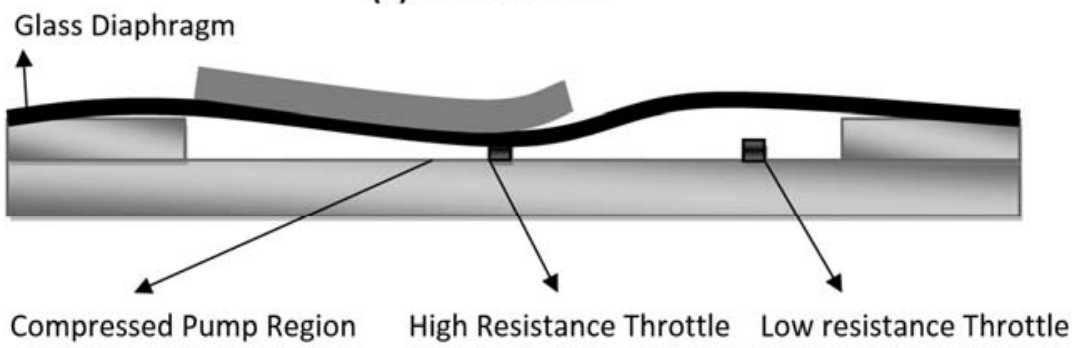

(c) Pumping Stroke

Fig. 4. Operation of a single piezoelectric micropump pumping from left to right (Adapted from [51]).

\section{1) Mechanical Micropumps}

Mechanical micropumps incorporate a physical actuator to accomplish the pumping action [42]. To regulate flow direction they may consist of a valve or incorporate valve less approach [45]. With mechanical micropumps, large flow rates can be achieved. Mechanical micropumps may use turbines to deliver constant volume of fluid in every pump cycle [46]. Most of the implantable micropumps are generally mechanical micropumps. Mechanical micropumps are further classified into electrostatic, piezoelectric, thermo-pneumatic, bimetallic, shape memory alloy (SMA), iconic conductive polymer film (ICPF).

\section{2) Non-mechanical Micropumps}

Non-mechanical micropumps do not rely on any physical actuation component hence their design and fabrication is much simpler than the mechanical micropumps. However, they are not as effective as mechanical micropumps when few factors like bulk pressure, flow rate and rise time response are considered moreover with these micropumps a much lower flow rate is realisable. Non-mechanical micropumps drive fluid into microchannels by converting non-mechanical energy into kinetic momentum. They can be driven by electrohydrodynamic (EHD), electrowetting, magnetohydrodynamic (MHD), phase transfer or electrochemical [42]. Among these, the electrochemically driven pump has lower power consumption, accurate flow rate and low heat generation [47]. 


\section{B. Microvalves}

Microvalves are the devices which control the fluid motion, its separation, timing and routeing in a microchannel [48] (See Fig. 5). They achieve it by varying a particular macroscopic parameter. These devices consist of a valve which can be an active valve or passive check valve. The time duration for which the valve remains activated is referred to as time constant which depends on the design [49]. For a particular micropump, the time constant is evaluated based on the specific application for which it is intended. The valves can be actuated by various methods and based on these methods five different categories of microvalves are electrokinetic, pneumatic, pinch, phase change and burst microvalve [48].

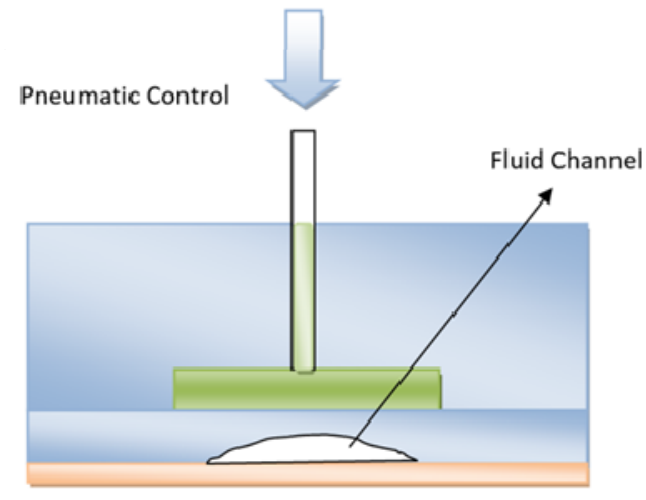

(a) Fluid apen channel
Pneumatic Control
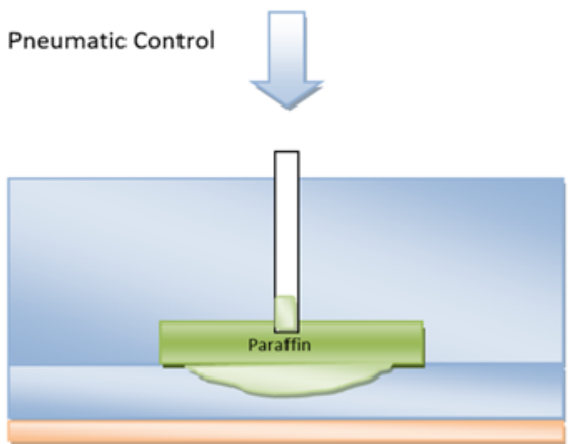

(b) Fluid Clased Channel

Fig. 5. Open and closed states of microvalve (Adapted from [52]).

TABLE I

Summary of MEMS Drug Delivery Techniques.

\begin{tabular}{|c|c|}
\hline $\begin{array}{l}\text { Transdermal } \\
\text { Drug Delivery }\end{array}$ & $\begin{array}{l}\text { Solid Durable Microneedles } \\
\text { - Application of drugs by patch or topical formulation on residual microholes } \\
\text { - Drugs are coated onto microneedles before applying them onto skin } \\
\text { Solid Degradable Microneedles } \\
\text { Hollow Microneedles }\end{array}$ \\
\hline $\begin{array}{l}\text { Oral Drug } \\
\text { Delivery }\end{array}$ & $\begin{array}{l}\text { Micropatches } \\
\text { - Flexible rate controlling membrane } \\
\text { - Adhesion to intestinal epithelium } \\
\text { - Multiple drug reservoirs } \\
\text { - Shortest diffusional path } \\
\text { - Fabricated using injection moulding } \\
\text { Bioadhesion } \\
\text { - Chemical approach - use of lectin as target molecule to enhance adhesion } \\
\text { - Physical approach - use of microposts, microneedles and nanostructures }\end{array}$ \\
\hline $\begin{array}{l}\text { Implantable } \\
\text { Devices for } \\
\text { Drug Delivery }\end{array}$ & $\begin{array}{l}\text { Micropump - Active drug delivery; Incorporate pumping action to dispense therapeutic } \\
\text { agents into body } \\
\text { - Mechanical - Rely on physical actuation; Complex Design } \\
\text { - Non-Mechanical - Don't rely on physical actuation; Simple Design } \\
\text { Microvalve - Incorporate valves which control the direction, timing and routing of drugs }\end{array}$ \\
\hline
\end{tabular}

\section{CONCLUSION}

This short and brief review summarises the current trends in drug delivery techniques which are facilitated by the use of MEMS technology, further, the benefits provided by MEMS techniques in the process of drug delivery are explored. In this review paper, a brief discussion of various microfabricated devices has been done and their usage for delivery of drugs in various ways has been discussed.

\section{REFERENCES}

[1] Sant, Shilpa, Sarah L. Tao, Omar Z. Fisher, Qiaobing Xu, Nicholas A. Peppas, and Ali Khademhosseini. "Microfabrication technologies for oral drug delivery." Advanced drug delivery reviews 64, no. 6 (2012): 496-507.

[2] Peppas, Nicholas A. "Intelligent therapeutics: biomimetic systems and nanotechnology in drug delivery." Advanced Drug Delivery Reviews 56, no. 11 (2004): 1529-1531.

[3] Santini, John T., Amy C. Richards, Rebecca A. Scheidt, Michael J. Cima, and Robert S. Langer. "Microchip technology in drug delivery." (2000): 377-379. 
[4] McAllister, Devin V., Mark G. Allen, and Mark R. Prausnitz. "Microfabricated microneedles for gene and drug delivery." Annual Review of Biomedical Engineering 2, no. 1 (2000): 289-313.

[5] R. H. Champion, J. L. Burton, and F. J. G. Ebling, Textbook of Dermatology. Boston: Blackwell Scientific, 1991.

[6] Henry, Sebastien, Devin V. McAllister, Mark G. Allen, and Mark R. Prausnitz. "Microfabricated microneedles: a novel approach to transdermal drug delivery." Journal of pharmaceutical sciences 87, no. 8 (1998): 922-925.

[7] Reed, Michael L., and W-K. Lye. "Microsystems for drug and gene delivery." Proceedings of the IEEE 92, no. 1 (2004): 56-75.

[8] Trimmer, William, Peter Ling, Chee-Kok Chin, Portia Orton, Randy Gaugler, Sarwar Hashmi, Ghazala Hashmi, Bruce Brunett, and Michael Reed. "Injection of DNA into plant and animal tissues with micromechanical piercing structures." In Proceedings of the IEEE Microelectromechanical Systems Workshop 8th, Amsterdam, pp. 111-115. 1995.

[9] Hilt, J. Zachary, and Nicholas A. Peppas. "Microfabricated drug delivery devices." International Journal of Pharmaceutics 306, no. 1 (2005): 15-23.

[10] Martanto, Wijaya, Shawn P. Davis, Nicholas R. Holiday, Jenny Wang, Harvinder S. Gill, and Mark R. Prausnitz. "Transdermal delivery of insulin using microneedles in vivo." Pharmaceutical research 21, no. 6 (2004): 947-952.

[11] Mikszta, John A., Jason B. Alarcon, John M. Brittingham, Diane E. Sutter, Ronald J. Pettis, and Noel G. Harvey. "Improved genetic immunization via micromechanical disruption of skin-barrier function and targeted epidermal delivery." Nature medicine 8 , no. 4 (2002): 415-419.

[12] Cormier, Michel, Bonny Johnson, Mahmoud Ameri, Kofi Nyam, Luz Libiran, Dee Dee Zhang, and Pete Daddona. "Transdermal delivery of desmopressin using a coated microneedle array patch system." Journal of controlled release 97, no. 3 (2004): 503-511.

[13] Matriano, James A., Michel Cormier, Juanita Johnson, Wendy A. Young, Margaret Buttery, Kofi Nyam, and Peter E. Daddona. "Macroflux ${ }^{\circledR}$ microprojection array patch technology: a new and efficient approach for intracutaneous immunization." Pharmaceutical research 19, no. 1 (2002): 63-70.

[14] Miyano, Takaya, Yoshikazu Tobinaga, Takahiro Kanno, Yasushi Matsuzaki, Hitoshi Takeda, Makoto Wakui, and Katsumi Hanada. "Sugar micro needles as transdermic drug delivery system." Biomedical Microdevices 7, no. 3 (2005): 185-188.

[15] Brazzle, John, Ian Papautsky, and A. Bruno Frazier. "Micromachined needle arrays for drug delivery or fluid extraction." IEEE engineering in medicine and biology magazine 18, no. 6 (1999): 53-58.

[16] Chandrasekaran, Shankar, John D. Brazzle, and A. Bruno Frazier. "Surface micromachined metallic microneedles." Journal of microelectromechanical systems 12 , no. 3 (2003): 281-288.

[17] Zahn, Jeffrey D., Neil H. Talbot, Dorian Liepmann, and Albert P. Pisano. "Microfabricated polysilicon microneedles for minimally invasive biomedical devices." Biomedical microdevices 2, no. 4 (2000): 295-303.

[18] Davis, Shawn P., Benjamin J. Landis, Zachary H. Adams, Mark G. Allen, and Mark R. Prausnitz. "Insertion of microneedles into skin: measurement and prediction of insertion force and needle fracture force." Journal of biomechanics 37, no. 8 (2004): 1155-1163.

[19] Staples, Mark, Karen Daniel, Michael J. Cima, and Robert Langer. "Application of micro-and nano-electromechanical devices to drug delivery." Pharmaceutical research 23, no. 5 (2006): 847-863.

[20] Chirra, Hariharasudhan D., and Tejal A. Desai. "Emerging microtechnologies for the development of oral drug delivery devices." Advanced drug delivery reviews 64, no. 14 (2012): 1569-1578.

[21] Colombo, P., F. Sonvico, G. Colombo, and R. Bettini. "Novel platforms for oral drug delivery." Pharmaceutical research 26, no. 3 (2009): 601-611.

[22] Colombo, Paolo, Patrizia Santi, Ruggero Bettini, Orazio Luca Strusi, Fabio Sonvico, and Gaia Colombo. "Modules, new assemblage kits and new assembles for the controlled release of substances." U.S. Patent Application 12/312,831, filed December 5, 2006.

[23] B Fox, Cade, Hariharasudhan D Chirra, and Tejal A Desai. "Planar bioadhesive microdevices: a new technology for oral drug delivery." Current pharmaceutical biotechnology 15, no. 7 (2014): 673-683.

[24] Tang, Rongbiao, Wei-Xia Li, Wei Huang, Fuhua Yan, Wei-Min Chai, Guo-Yuan Yang, and Ke-Min Chen. "CO2-based in-line phase contrast imaging of small intestine in mice." Scientific reports 3 (2013): 2313.

[25] Tao, Sarah L., and Tejal A. Desai. "Gastrointestinal patch systems for oral drug delivery." Drug discovery today 10, no. 13 (2005): 909-915.

[26] Tao, Sarah L., and Tejal A. Desai. "Microfabrication of multilayer, asymmetric, polymeric devices for drug delivery." Advanced Materials 17, no. 13 (2005): 1625-1630.

[27] Tao, Sarah L., and Tejal A. Desai. "Micromachined devices: the impact of controlled geometry from cell-targeting to bioavailability." Journal of Controlled Release 109, no. 1 (2005): 127-138.

[28] Ainslie, Kristy M., and Tejal A. Desai. "Microfabricated implants for applications in therapeutic delivery, tissue engineering, and biosensing." Lab on a Chip 8, no. 11 (2008): 1864-1878.

[29] Lu, Y., and S. C. Chen. "Micro and nano-fabrication of biodegradable polymers for drug delivery." Advanced drug delivery reviews 56, no. 11 (2004): 1621-1633.

[30] Guan, Jingiiao, Hongyan He, L. James Lee, and Derek J. Hansford. "Fabrication of particulate Reservoir - Containing, capsulelike, and Self - Folding polymer microstructures for drug delivery." Small 3, no. 3 (2007): 412-418.

[31] Serra, Laura, Josep Doménech, and Nicholas A. Peppas. "Engineering design and molecular dynamics of mucoadhesive drug delivery systems as targeting agents." European journal of pharmaceutics and biopharmaceutics 71, no. 3 (2009): 519-528.

[32] Duchěne, D., F. Touchard, and N. A. Peppas. "Pharmaceutical and medical aspects of bioadhesive systems for drug administration." Drug Development and Industrial Pharmacy 14, no. 2-3 (1988): 283-318.

[33] Lee, Jeong W., Jung-Hwan Park, and Mark R. Prausnitz. "Dissolving microneedles for transdermal drug delivery." Biomaterials 29, no. 13 (2008): 2113-2124.

[34] Park, Jung-Hwan, Mark G. Allen, and Mark R. Prausnitz. "Polymer microneedles for controlled-release drug delivery." Pharmaceutical research 23, no. 5 (2006): 1008-1019.

[35] Spolenak, Ralph, Stanislav Gorb, and Eduard Arzt. "Adhesion design maps for bio-inspired attachment systems." Acta Biomaterialia 1, no. 1 (2005): 5-13.

[36] Zhang, Sam, Deen Sun, Yongqing Fu, and Hejun Du. "Toughening of hard nanostructural thin films: a critical review." Surface and Coatings Technology 198, no. 1 (2005): 2-8.

[37] Fischer, Kathleen E., Benjamin J. Alemán, Sarah L. Tao, R. Hugh Daniels, Esther M. Li, Mark D. Bünger, Ganesh Nagaraj, Parminder Singh, Alex Zettl, and Tejal A. Desai. "Biomimetic nanowire coatings for next generation adhesive drug delivery systems." Nano letters 9, no. 2 (2009): 716-720.

[38] DeSesso, John M., and Amy Lavin Williams. "Contrasting the gastrointestinal tracts of mammals: factors that influence absorption." Annual reports in medicinal chemistry 43 (2008): 353-371.

[39] Li, Yawen, Rebecca S. Shawgo, Betty Tyler, Paul T. Henderson, John S. Vogel, Aron Rosenberg, Phillip B. Storm, Robert Langer, Henry Brem, and Michael J. Cima. "In vivo release from a drug delivery MEMS device." Journal of Controlled Release 100, no. 2 (2004): 211-219. 
[40] Tng, Danny Jian Hang, Rui Hu, Peiyi Song, Indrajit Roy, and Ken-Tye Yong. "Approaches and challenges of engineering implantable microelectromechanical systems (MEMS) drug delivery systems for in vitro and in vivo applications." Micromachines 3, no. 4 (2012): 615-631.

[41] Elman, N. M., HL Ho Duc, and M. J. Cima. "An implantable MEMS drug delivery device for rapid delivery in ambulatory emergency care." Biomedical microdevices 11, no. 3 (2009): 625-631.

[42] Tsai, Nan-Chyuan, and Chung-Yang Sue. "Review of MEMS-based drug delivery and dosing systems." Sensors and Actuators A: Physical 134, no. 2 (2007): 555-564.

[43] Kotzar, Geoffrey, Mark Freas, Phillip Abel, Aaron Fleischman, Shuvo Roy, Christian Zorman, James M. Moran, and Jeff Melzak. "Evaluation of MEMS materials of construction for implantable medical devices." Biomaterials 23, no. 13 (2002): 2737-2750.

[44] Huang, Xiaoyang, and Toh Kok Chuan. "MEMS-micropumps: a review." In Journal of Fluids Engineering-Transactions of the ASME. 2002.

[45] [45] Stemme, Erik, and Göran Stemme. "A valveless diffuser/nozzle-based fluid pump." Sensors and Actuators A: physical 39, no. 2 (1993): $159-167$.

[46] White, F. M. "Fluid Mechanics, 732 pp." (1986).

[47] Böhm, Sebastian, Björn Timmer, Wouter Olthuis, and Piet Bergveld. "A closed-loop controlled electrochemically actuated microdosing system." Journal of Micromechanics and Microengineering 10, no. 4 (2000): 498.

[48] Au, Anthony K., Hoyin Lai, Ben R. Utela, and Albert Folch. "Microvalves and micropumps for BioMEMS." Micromachines 2, no. 2 (2011): 179-220.

[49] Oh, Kwang W., and Chong H. Ahn. "A review of microvalves." Journal of micromechanics and microengineering 16, no. 5 (2006): R13.

[50] Arora, Anubhav, Mark R. Prausnitz, and Samir Mitragotri. "Micro-scale devices for transdermal drug delivery." International journal of pharmaceutics 364 , no. 2 (2008): 227-236.

[51] Tracey, M. C., I. D. Johnston, J. B. Davis, and C. K. L. Tan. "Dual independent displacement-amplified micropumps with a single actuator." Journal of Micromechanics and Microengineering 16, no. 8 (2006): 1444.

[52] Yang, Bozhi, and Qiao Lin. "A latchable microvalve using phase change of paraffin wax." Sensors and Actuators A: Physical 134, no. 1 (2007): 194-200.

\section{AUTHOR PROFILE}

Mohammad Sibgat Ullah, Rishab Kaw, Peer Athar Rasool are Senior Undergraduates in DoECE, SMVDU, India - 182320. Their research interests include MEMS, VLSI and nanotechnology and their applications that can make the world a better place.

Mr. Neeraj Tripathi is working as Assistant Professor in the Department of Electronics and Communication Engineering at Shri Mata Vaishno Devi University. He has completed B.E in Electronics and Communication Engineering from Madan Mohan Malaviya Engineering College, Gorakhpur followed by M.Tech(Microelectronics and VLSI Design) from Indian Institute of Technology, Kharagpur(IIT, Kharagpur). Mr. Neeraj Tripathi has also completed Professional Degree of Second Level in NEMS/MEMS from University of Trento, Italy. He initiated the course on the MEMS at undergraduate level and post graduate level at SMVDU, Katra. He has vast teaching experience in the field of VLSI, Microelectronics, MEMS, IC fabrication both at undergraduate and post graduate level. Prior to joining SMVDU, he has worked in several universities. He did research at the University of Trento, Italy in the field of radiation micro detectors. He has published several papers in peer- reviewed journals and conferences.

His present research areas include TCAD Modeling, Neuroprosthetics, Implantable microelectrodes and devices, BMI, stimulation, and recording of brains, VLSI, Semiconductor modeling, Multiphysics modeling.

Mr. Vikram Singh did B.Tech in Electronics and Communication Engineering from Kurukshetra University, India in 2004. He did M.Tech (VLSI Design and Embedded Systems) from Guru Jambheshwr University of Science and Technology, India in 2008. He has more than 8 years of teaching experience and 1 year industrial experience. He has published more than 12 papers in peer reviewed journals/conferences. He is pursuing $\mathrm{Ph} . \mathrm{D}$ in Low Power RF Integrated circuits. His research arear includes VLSI Design, MEMS, IC Design, CMOS Analog Integrated circuits etc. At present he is working as Assistant Professor in the Department of Electronics and Communication Engineering at Shri Mata Vaishno Devi University, Katra (J\&K). 